
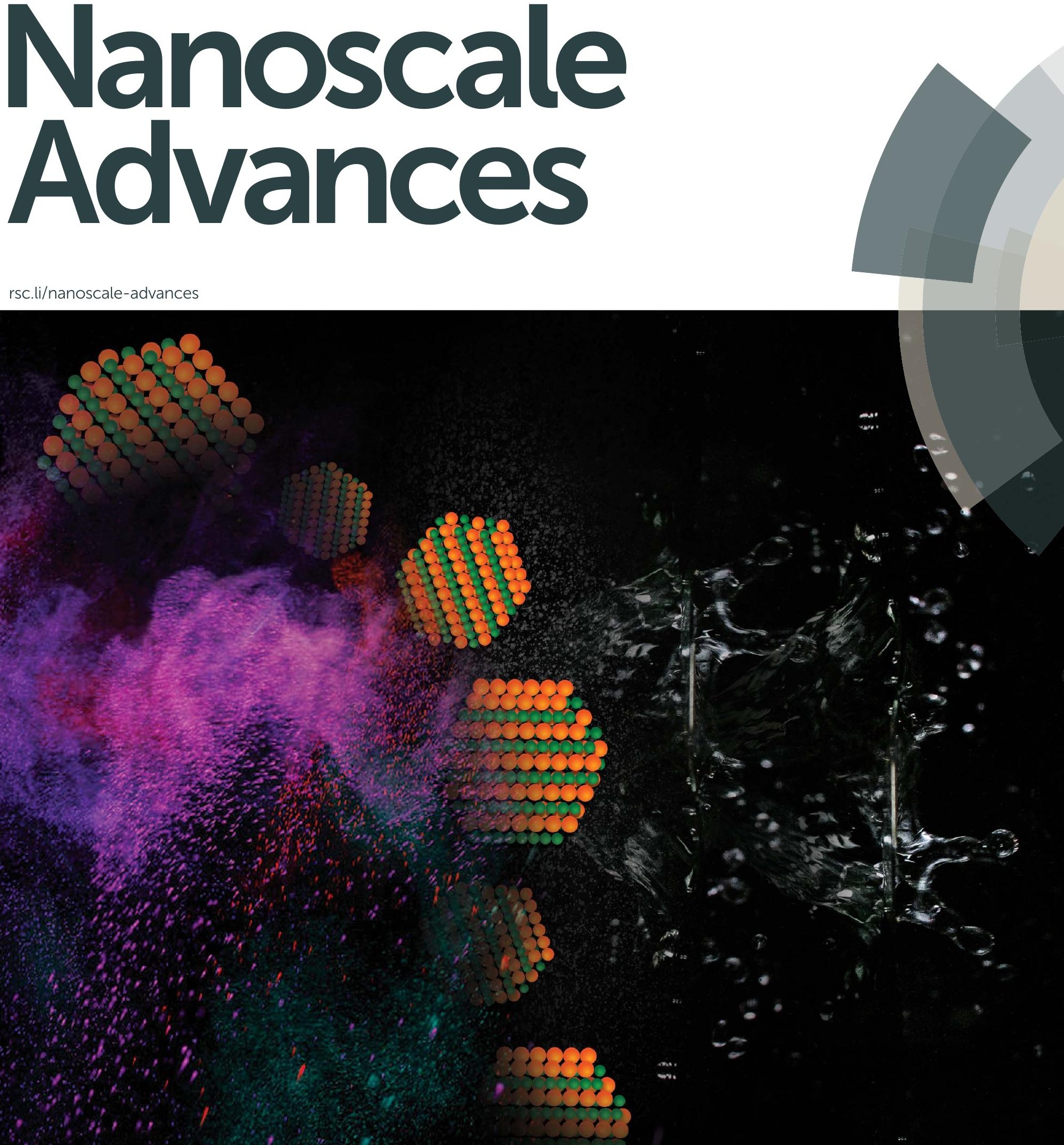

ISSN 2516-0230

ROYAL SOCIETY | Celebrating OF CHEMISTRY | IYPT 2019
PAPER

Marc R. Knecht et al.

Biomimetic strategies to produce catalytically reactive CuS nanodisks 
Check for updates

Cite this: Nanoscale Adv., 2019, 1, 2857

\title{
Biomimetic strategies to produce catalytically reactive CuS nanodisks $\dagger$
}

\author{
Elise C. Bell, $\hbar^{\mathrm{a}}$ Catherine J. Munro,,$^{\mathrm{a}}$ Joseph M. Slocik, ${ }^{\mathrm{b}}$ Dharmendra Shukla, ${ }^{\mathrm{c}}$ \\ Atul D. Parab, ${ }^{a}$ Joshua L. Cohn (D) ${ }^{c}$ and Marc R. Knecht (iD *ad
}

Copper sulfide materials have diverse applications from cancer therapy to environmental remediation due to their narrow bandgap and easily tuned plasmon. The synthesis of these materials often involves toxic reagents and harsh conditions where biomimetic methods may provide opportunities to produce these structures under sustainable conditions. To explore this capability, simple amino acids were exploited as biological ligands for the ambient synthesis of CuS materials. Using an aqueous-based approach, CuS nanodisks were prepared using acid-containing amino acid molecules that stabilize the materials against bulk aggregation. These structures were fully characterized by UV-vis analysis, transmission electron microscopy, dynamic light scattering, atomic force microscopy, selected area electron diffraction, and $\mathrm{X}$-ray diffraction, which confirmed the formation of CuS. The materials possessed a vibrant plasmon band in the near IR region and demonstrated enhanced photocatalytic reactivity for the advanced oxidation of organic dyes in water. These results demonstrate a room temperature synthetic route to optically important materials, which could have important application in catalysis, optics, nanomedicine, etc.

Received 28th May 2019

Accepted 11th June 2019

DOI: $10.1039 / c 9 n a 00335 \mathrm{e}$

rsc.li/nanoscale-advances require unsustainable conditions. ${ }^{\mathbf{1 7 - 2 0}}$ In this regard, CuS production typically requires harsh conditions, toxic organic solvents, ${ }^{21}$ and/or complex instrumentation that can limit their long-term production. For instance, Chaudhuri and coworkers generated a library of CuS materials of varying shape and composition by using different ratios of chelating and nonchelating solvents in a solvothermal process. Here, $\mathrm{Cu}\left(\mathrm{NO}_{3}\right)_{2}$ and thiourea were put into a Teflon-lined stainless steel autoclave with ethanol, ethylenediamine, and/or ethyleneglycol. These contents were sealed and maintained at $130{ }^{\circ} \mathrm{C}$ for $12 \mathrm{~h}$ prior to being washed with water/absolute ethanol and then dried in a vacuum oven at $60{ }^{\circ} \mathrm{C}$ for $3 \mathrm{~h} .{ }^{21}$ Others have used templates $^{22}$ or top-down ${ }^{23}$ synthetic methods to achieve desired CuS materials. Wu et al. established a large-scale synthesis of CuS nanotubes in a two-step process where sacrificial copper wires were first made in the presence of $\mathrm{NaOH}$, ethylenediamine, and hydrazine. These wires were washed and dried before being sonicated at $80{ }^{\circ} \mathrm{C}$ for $12 \mathrm{~h}$ with ethyleneglycol and thiourea. ${ }^{22}$

As an alternative to traditional synthetic approaches, biomolecules have been widely used for the production of inorganic nanostructures due to their inherent ability to stabilize growing nanoparticle surfaces through non-covalent interactions. ${ }^{24,25}$ In general, such approaches entail processing under ambient conditions in aqueous solutions at room temperature and pressure. Such biomimetic approaches have been exploited to generate materials for specific applications including catalysis,${ }^{26,27}$ nanomedicine, ${ }^{28}$ plasmonics,${ }^{29,30}$ etc. While bio-inspired 
methods have been applied to produce $\mathrm{ZnS}^{31,32}$ and $\mathrm{CdS},{ }^{32,33}$ few biomimetic methods have been used in the synthesis of CuS. For instance, da Costa et al. exploited sulfide from sulfate reducing bacteria to generate spherical CuS particles of $\sim 3 \mathrm{~nm}$ in diameter. ${ }^{34}$ More recently, Ortiz-Ramos et al. have grown CuS films using alanine, glycine, and serine as complexing agents. ${ }^{35}$

While these bio-based methods result in the production of CuS materials, they generally rely on moderate temperatures $\left(>40^{\circ} \mathrm{C}\right)$ and $\mathrm{pH}$ conditions to achieve material formation. More information is required to advance the biomimetic production of CuS nanoparticles under sustainable, eco-friendly conditions (e.g. in water, on the benchtop, at room temperature). ${ }^{36-44}$ This requires a foundation in understanding how simple small biomolecules (e.g. amino acids) stabilize the materials, which remains generally unclear.

Herein we describe a sustainable approach to produce CuS nanodisks using acidic amino acids to passivate and stabilize the inorganic materials. The materials were fabricated through the reaction of $\mathrm{Cu}^{2+}$ and $\mathrm{S}^{2-}$ in the presence of aspartic acid (Asp) or glutamic acid (Glu). Once prepared, the materials were fully characterized by transmission electron microscopy (TEM), including high-resolution TEM (HR-TEM), selected area electron diffraction (SAED), UV-vis near infrared (NIR) and diffuse reflectance (DRS) spectroscopies, powder X-ray diffraction (XRD), and dynamic light scattering (DLS). Interestingly, a change in material structure as a function of the amino acid passivant was observed after sample lyophilization, which was required to probe the photocatalytic properties of the materials. The dried materials were studied for the photocatalytic degradation of three model organic dyes using Fenton type catalytic processes, which revealed reactivity differences between the two different structures. Taken together, these studies demonstrated the production of important optically active materials for both plasmonics and photocatalytic reactions, where their stability and properties are dictated by the biomimetic surface passivant used during material production.

\section{Experimental}

\section{Chemicals}

L-Aspartic acid and L-glutamic acid were obtained from SigmaAldrich. Neutral red, methyl orange, methylene blue, $\mathrm{CuCl}_{2}$ $\cdot 2 \mathrm{H}_{2} \mathrm{O}$, and $\mathrm{Na}_{2} \mathrm{~S}$ were purchased from Alfa Aesar. Finally, $35 \mathrm{wt} \%$ hydrogen peroxide was purchased from VWR. All chemicals were used as received without further purification. Milli-Q water (18 $\mathrm{M} \Omega \mathrm{cm}$ ) was used for all experiments.

\section{Synthesis of CuS}

In a $25.0 \mathrm{~mL}$ vial, $3.0 \mathrm{~mL}$ of $0.01 \mathrm{M}$ Asp, $1.0 \mathrm{~mL}$ of $0.01 \mathrm{M}$ $\mathrm{CuCl}_{2} \cdot 2 \mathrm{H}_{2} \mathrm{O}$, and $6.0 \mathrm{~mL}$ of $\mathrm{H}_{2} \mathrm{O}$ were added. The resulting solution was stirred for $15 \mathrm{~min}$, followed by addition of $0.3 \mathrm{~mL}$ of $0.1 \mathrm{M} \mathrm{Na}_{2} \mathrm{~S}$ and $9.7 \mathrm{~mL}$ of $\mathrm{H}_{2} \mathrm{O}$. This reaction solution results in a $\mathrm{Cu}^{2+}: \mathrm{S}^{2-}$ ratio of $1: 3$. The solution was subsequently stirred continuously for $24 \mathrm{~h}$ on the benchtop at room temperature. Upon addition of $\mathrm{Na}_{2} \mathrm{~S}$ to the reaction mixture, the solution immediately turned burnt orange then slowly faded to a dark green color over the $24 \mathrm{~h}$ process. After stirring, the materials were lyophilized, resulting in a fine black powder that was used for catalysis.

\section{Material characterization}

Low-resolution TEM analysis was performed using a JEOL JEM2010 microscope operating at $80 \mathrm{kV}$, while HR-TEM studies were completed using an FEI $\mathrm{C}_{\mathrm{s}}$-corrected Titan TEM microscope operating at $300 \mathrm{kV}$. Selected area electron diffraction was obtained using spot size 8 and a camera length of $360 \mathrm{~mm}$. The sample was prepared by drop-casting $10.0 \mu \mathrm{L}$ of the CuS sample onto a carbon-coated 400 mesh $\mathrm{Cu}$ grid (EM Sciences), which was dried overnight. AFM samples were prepared in a similar fashion. For this, $50.0 \mu \mathrm{L}$ of the CuS sample was drop cast on to a clean silicon surface and allowed to settle for $\sim 2-3 \mathrm{~h}$. After this time, the remaining solution was removed and the surface was dried overnight prior to analysis using Digital Instruments/ Veeco AFM (Dimension 3100).

The as prepared CuS materials were studied on a Cary 5000 UV-vis-NIR spectrophotometer using a quartz cuvette with a $1.0 \mathrm{~cm}$ path length. UV-vis DRS analysis of the solid materials was completed on a Shimadzu Model UV-2600 system. The lyophilized CuS sample was put into a clean $2.0 \mathrm{~mm}$ quartz cuvette with $\sim 600 \mathrm{mg}$ of the CuS so that when the sample was packed into the cuvette, it was at least $2 / 3$ full. The spectrum of the sample was taken and converted into a Tauc plot before being analyzed using the Kubelka-Munk function, $F\left(R_{\infty}\right)$, where the band gap values can be obtained from the tangent line of the Tauc plot.

Powder XRD analysis was completed on a Philips MRD X'Pert diffractometer using $\mathrm{Cu} \mathrm{K} \alpha$ radiation. Samples were prepared on ozone cleaned glass slides, where at least $1 \mathrm{~cm}^{2}$ was covered in a thin spread of vacuum grease before an even layer of the CuS powdered material was added to the slide and analyzed. Finally, for DLS analysis, the as prepared CuS materials were inserted into a disposable folded capillary cell and run on a Malvern Nano Series Nano-ZS to elucidate the surface charge of the CuS materials.

\section{Fenton dye degradation protocol}

Degradation of neutral red, methyl orange, and methylene blue was done using an advanced oxidation process in the presence of $\mathrm{H}_{2} \mathrm{O}_{2}$ employing modified methods. ${ }^{23}$ Briefly, $10.0 \mathrm{mg}$ of the lyophilized CuS material was suspended in $1.0 \mathrm{~mL}$ of water and sonicated for $5 \mathrm{~min}$ before $206.0 \mu \mathrm{L}$ of the suspension was added to $1.38 \mathrm{~mL}$ of $125 \mathrm{mg} \mathrm{L}^{-1}$ dye solution in a $1.0 \mathrm{~cm}$ quartz cuvette. This mixture was stirred in the dark for $1 \mathrm{~h}$ to allow for dye adsorption to the material surface. Next, $412.0 \mu \mathrm{L}$ of $35 \mathrm{wt} \%$ $\mathrm{H}_{2} \mathrm{O}_{2}$ was added to the cuvette under constant stirring. This reaction solution was immediately exposed to a $1000 \mathrm{~W}$ Xe arc lamp operating at $\sim 100 \mathrm{~mW} \mathrm{~cm} \mathrm{~cm}^{-2}$ in an Oriel Sol1A Class ABB solar simulator. The sample to light source distance was $\sim 10 \mathrm{~cm}$. The cuvette holding the reaction was analyzed at least every $15 \mathrm{~min}$ on an Agilent 8453 spectrophotometer where the dye absorbance was tracked at the $\lambda_{\max }$. For recyclability, an additional $172.0 \mu \mathrm{L}$ of $1.0 \mathrm{~g} \mathrm{~L}^{-1}$ dye solution and $412.0 \mu \mathrm{L}$ of 
$\mathrm{H}_{2} \mathrm{O}_{2}$ were added to the reaction mixture and immediately exposed to the lamp source. This was done to re-establish the catalyst to substrate ratio in the initial degradation cycle. Note there is an increase in volume without the loss of catalyst for each recycle analysis due to the available concentration of $\mathrm{H}_{2} \mathrm{O}_{2}$.

\section{Results and discussion}

Biomimetic production of CuS nanomaterials was examined using a selection of amino acids based upon their side chain composition. Gly and Val were selected as aliphatic amino acids, while Tyr was used to represent aromatic side chains. Both Cys and Met were used due to their sulfur containing group, while Gln was tested to examine the effects of the amidebased amino acids. Finally, basic amine-containing Arg, His, and Lys were tested, while Asp and Glu were used because of their acid-containing side chains. To prepare the CuS materials, a simple one pot method was employed. For this, $\mathrm{Cu}^{2+}$ ions were commixed with the selected amino acid in water, followed by the addition of $\mathrm{Na}_{2} \mathrm{~S}$. The reaction was subsequently allowed to stir for $24 \mathrm{~h}$ on the bench top to facilitate material production. Substantial differences in the reactions were observed based upon the amino acid in the mixture. For all the biomolecules studied, only Glu and Asp prepared stable materials over $24 \mathrm{~h}$ that presented a plasmon band; for the reactions in the presence of all other amino acids, no evidence of material production was observed and/or the materials precipitated after $\sim 4 \mathrm{~h}$. As such, all further studies were only processed with Asp and Glu.

Fig. 1 presents the UV-vis-NIR analysis of the material synthesis using Asp and Glu. Note that due to the absorbance of water from 1360 to $1600 \mathrm{~nm}$, no data is presented in that window. Fig. 1a specifically presents the analysis for the materials prepared using Asp. The absorbance of the reaction mixture after each component addition demonstrated no absorbance over the region of interest; however, after $24 \mathrm{~h}$ of reaction, formation of a strong plasmon band at $1300 \mathrm{~nm}$ was observed. Such a peak position is consistent with the formation of CuS-based materials that are known to have a strong absorbance in the NIR. ${ }^{\mathbf{1 2 , 1 3 , 4 5 - 4 7}}$ Such a change in optical properties is consistent with changes in solution color that present as dark orange immediately after $\mathrm{S}^{2-}$ addition and evolve to dark green after $24 \mathrm{~h}$ of reaction. Identical results were noted for the system prepared in the presence of Glu (Fig. 1b) suggesting that the material growth process is quite similar, regardless of which acid-containing amino acid is employed.

Fig. 2 displays the morphology of the as prepared CuS materials by low-resolution TEM, where the inset is a zoomed in portion of the image. As is evident, both systems generated nanodisks of CuS materials. Statistical analysis of at least 100 particles indicated that the structures prepared using Asp demonstrated an average size $48.5 \pm 14.7 \mathrm{~nm}$, while the Glubased materials presented an average size of $32.1 \pm 10.3 \mathrm{~nm}$. Fig. S2 in the ESI† presents the particle size histograms of the two materials. Such values are similar to the sizes determined by DLS for the Asp- and Glu-passivated materials, which indicated hydrodynamic diameters of $30.2 \pm 5.6$ and $30.7 \pm 6.6 \mathrm{~nm}$,
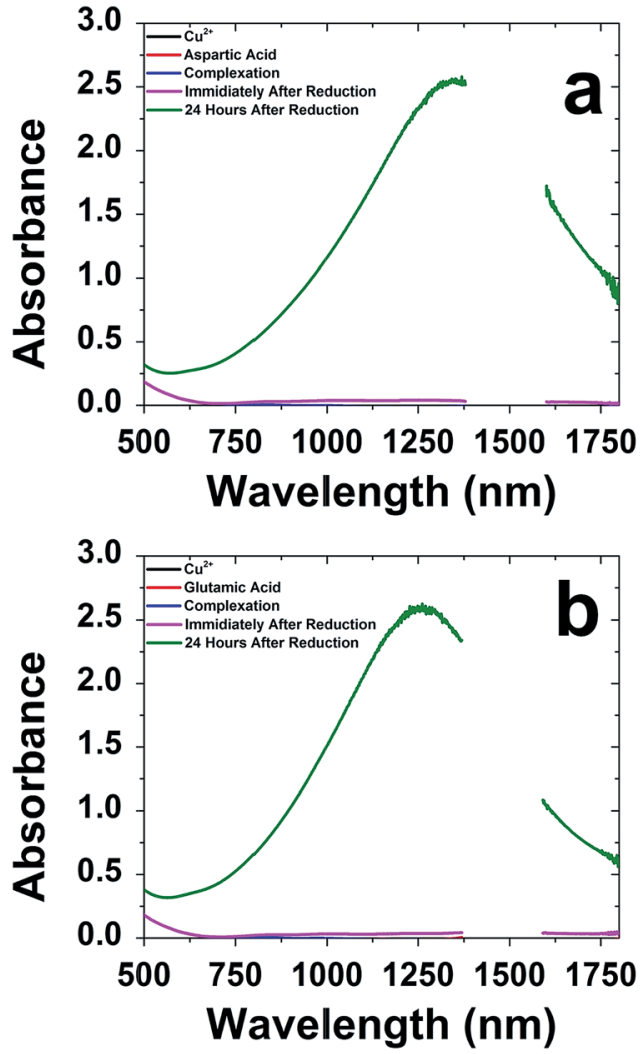

Fig. 1 UV-vis-NIR analysis of CuS materials produced in the presence of (a) Asp and (b) Glu.

respectively. Note that zeta potential analysis of these nanodisks indicated a surface charge of $-18.1 \pm 2.1 \mathrm{mV}$ for the Asp-capped materials and $-40.9 \pm 2.4 \mathrm{mV}$ for the Glu-passivated structures.

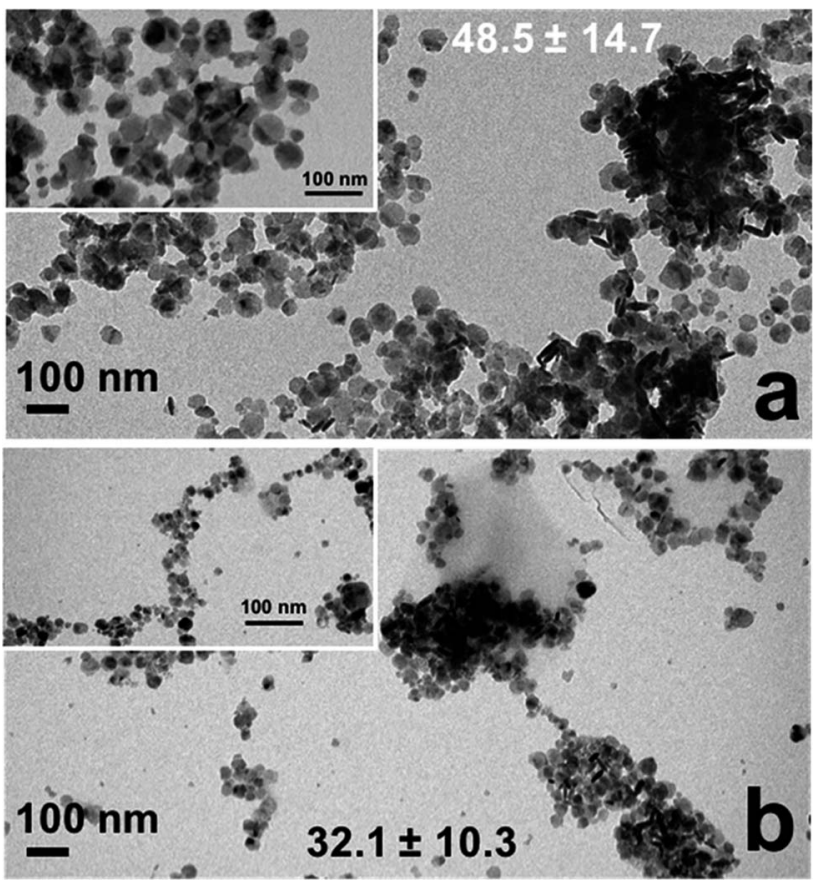

Fig. 2 TEM analysis of the CuS nanodisks prepared using (a) Asp and (b) Glu. The inset shows a zoomed in region of the materials. 
This potentially suggests enhanced amino acid adsorption in the presence of Glu to give rise to the larger negative change, which may affect the catalytic properties of the materials.

Additional imaging and analysis of the CuS materials were obtained, including HR-TEM and AFM. From the HR-TEM analysis (Fig. 3), both the Asp- and Glu-based nanodisks were highly crystalline, as anticipated. Clear lattice fringes of $\sim 0.28 \mathrm{~nm}$ were observed for both samples, corresponding to the (103) lattice of CuS. This crystallinity is supported by the SAED analysis. For both materials (Fig. $3 \mathrm{~b}$ and d), the SAED patterns of the as prepared nanodisks demonstrated diffraction rings consistent with the (101), (102), (103), (110), (108), and (116) indices for CuS (JCPDS: 01-078-0876).

While the TEM analysis can observe the lateral dimensions of the CuS nanodisks, it cannot measure the absolute height of the material. To ascertain this level of information, the CuS structures were imaged using AFM. For this, the materials were deposited onto a silicon surface and allowed to settle before excess solvent was wicked away and the surface dried overnight, followed by AFM imaging. As shown in Fig. 4a, the Asp-capped CuS nanostructures demonstrated their disk-like morphology. Tracking the height profile of the materials for this sample (Fig. 4b), they varied between 1 and $2 \mathrm{~nm}$ in thickness. Such a value is substantially smaller than the lateral dimensions, confirming the disk-like morphology. Fig. 4c presents the AFM analysis of the Glu-prepared CuS materials, which were similar to the Asp-based materials; however, they tended to be thicker in dimension with heights ranging between 1.5 and $4 \mathrm{~nm}$.

Although it is evident that CuS nanodisks were prepared using both Asp and Glu, based upon the TEM and AFM imaging studies, notable differences in material dimensions were

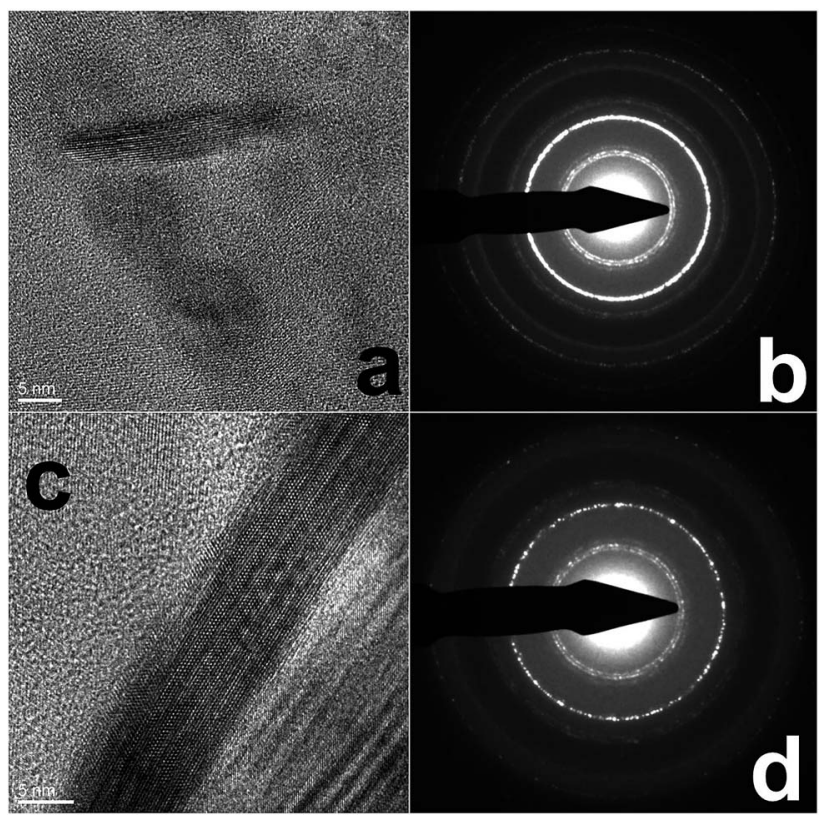

Fig. 3 HR-TEM and SAED analyses of the CuS materials prepared using ( $a$ and $b$ ) Asp and ( $c$ and d) Glu. Parts ( $a$ and $c$ ) displays the HRTEM images, while parts ( $b$ and $d$ ) present the SAED patterns of the corresponding materials.
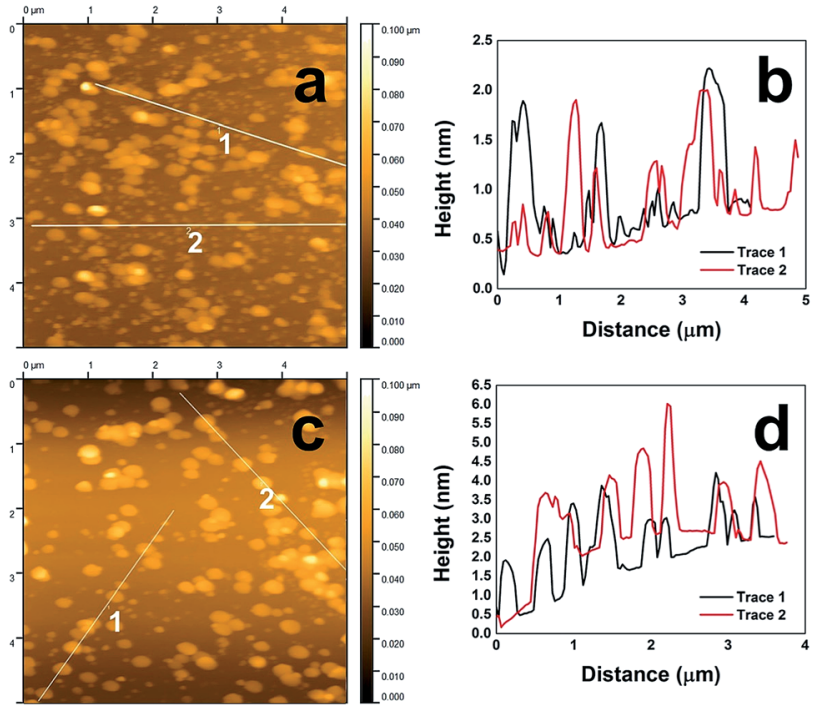

Fig. 4 AFM analysis of the CuS materials prepared using ( $a$ and b) Asp and ( $c$ and d) Glu. The AFM image of the materials is shown in parts (a and $\mathrm{c}$ ), while the corresponding height profile is presented in parts (b and d).

evident. In this regard, the lateral dimension for the Asp-based structures was greater than that of the Glu-prepared materials; however, the Asp-capped CuS were thinner in height as compared to the Glu-stabilized sample. This suggests that specific differences in material growth are likely to occur, arising potentially from the affinity of the different amino acids for the CuS surface. These differences could result in changes in material stability and could affect their overall reactivity.

To prepare the CuS nanodisks for further analyses and for catalytic studies, the materials were lyophilized to remove the water solvent and stored in a vacuum oven at $60{ }^{\circ} \mathrm{C}$ for at least $24 \mathrm{~h}$. To understand the potential changes in material morphology, the dried powders were resuspended in water and drop casted for additional TEM analysis (Fig. 5). TEM images of the dried Asp-stabilized material are presented in Fig. 5a and b, which indicate that the nanodisks collapsed into spherical particles. The average size of the new spherical materials was $5.8 \pm 3.1 \mathrm{~nm}$. TEM images of the Glu-based structures after

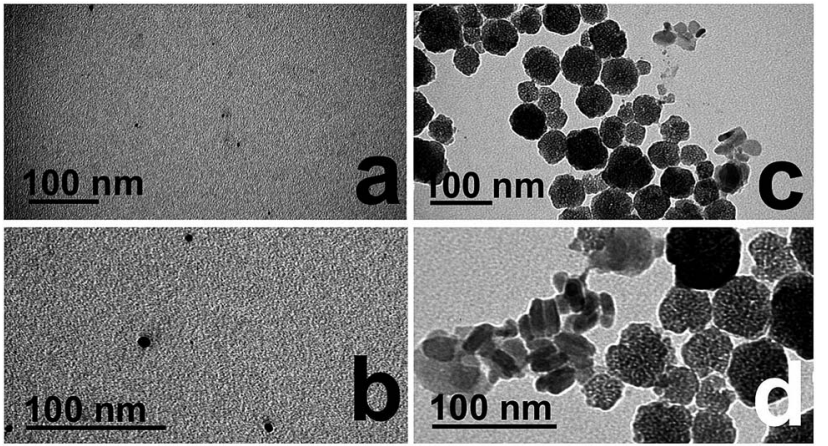

Fig. 5 TEM analysis of the ( $a$ and b) Asp- and ( $c$ and d) Glu-capped CuS materials after lyophilization to a powder. 
drying are presented in Fig. 5c and d. Remarkably, these materials retained their disk-like morphology; however, their average diameter increased to $85.7 \pm 54.8 \mathrm{~nm}$.

To confirm the structure of the dried materials, powder XRD was employed of the lyophilized samples (Fig. 6). The diffraction pattern presented by the Asp- and Glu-passivated materials was analyzed and the peaks were labeled using literature values. ${ }^{48,49}$ Both materials display peaks at $27.7^{\circ}, 29.3^{\circ}, 31.9^{\circ}$, $48.1^{\circ}, 52.4^{\circ}$, and $59.0^{\circ}$, corresponding to (101), (102), (103), (110), (108), and (116) diffraction planes of CuS, respectively. These peak patterns closely represent the covellite form of CuS due to the presence of the (102) plane at $29.3^{\circ}$ and the (110) plane at $48.1^{\circ}$, both of which disappear when digenite $\left(\mathrm{Cu}_{1.8} \mathrm{~S}\right)$ and chalcocite $\left(\mathrm{Cu}_{1.96} \mathrm{~S}\right)$ become more prevalent in the crystal. $^{48-50}$ Such diffraction patterns are also fully consistent with the SAED patterns, confirming the formation of the covellite structure of CuS.

As a final characterization method, UV-vis DRS was performed to characterize the optical properties of the materials to quantify the band gap of the structures in the solid state. Fig. 7a shows the diffuse reflectance absorbance spectra for both material sets, while Fig. $7 \mathrm{~b}$ and c present the Tauc plots of Aspand Glu-prepared materials, respectively. Based upon the slope of the tangent line, the Asp-based material has a band gap of $2.56 \mathrm{eV}$, while Glu-prepared structures have a band gap of $2.54 \mathrm{eV}$. These values are consistent with bulk CuS of the hexagonal wurtzite structure that has an approximate band gap of $2.5 \mathrm{eV} .^{10}$

From the results presented above, only the acidic amino acids (Glu and Asp) are able to make stable CuS materials. Using these amino acids, the fabrication of CuS nanodisks occurs, where the other biomolecules used led either to material precipitation or the lack of particle formation. This suggests that the acidic side chains are important for coordination to the

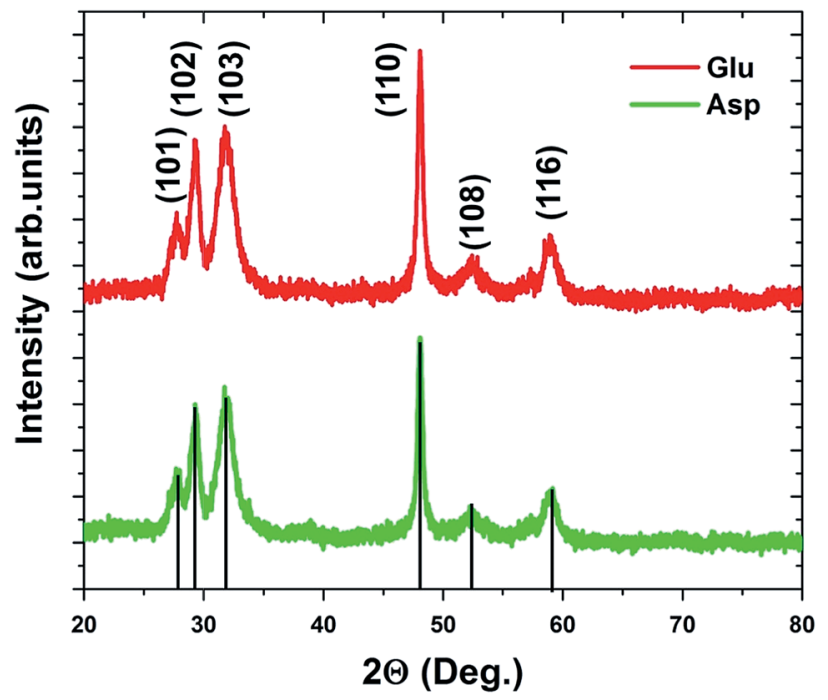

Fig. 6 Powder XRD analysis of the Asp- and Glu-stabilized CuS materials after lyophilization displaying diffractions associated with the covellite form of CuS.
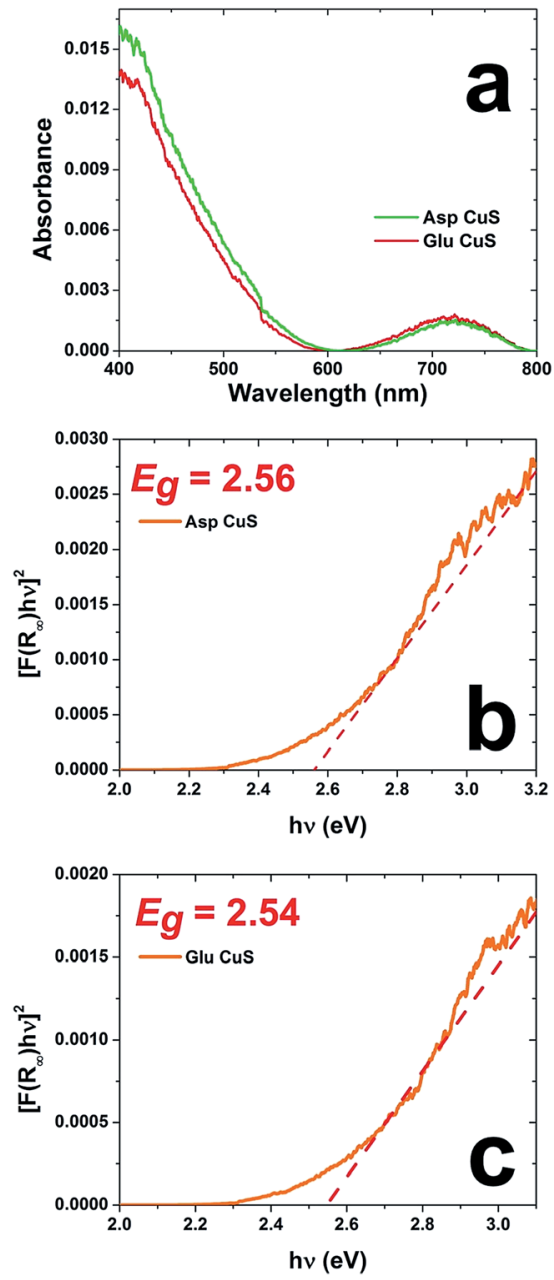

Fig. 7 UV-vis DRS analysis of the CuS materials. Part (a) presents the DRS spectra of the Asp- and Glu-capped CuS, while the Tauc plots for the materials are presented in parts (b and c), respectively.

growing CuS surface to prevent particle aggregation and facilitate the formation of a stable colloidal suspension. In the work of Nelwamondo et al. it is proposed that deprotonated Ala has enhanced affinity for $\mathrm{Cu}^{2+}$ cations in solution, driving the stability of the complex prior to material formation. ${ }^{51}$ This explanation may justify why Asp and Glu were effective under our conditions. Asp has a $\mathrm{p} K_{\mathrm{a}}$ value of 3.71 while Glu has a $\mathrm{p} K_{\mathrm{a}}$ value of 4.15. The $\mathrm{pH}$ of both syntheses was taken $1 \mathrm{~h}$ after addition of the NaS and were found to be 6.58 and 6.79, respectively. At these $\mathrm{pH}$ values both residues would exist in their anionic form; however, this $\mathrm{pH}$ is too low to establish the anionic form of the other amino acids studied. This potentially identifies the basis of the acidic residues in their ability to stabilize the CuS materials; however, additional factors beyond molecular charge are probable.

Once the materials were dried into the solid state, their structure changed, either to form nanoparticles (Asp) or larger nanodisks (Glu). Such results suggest that the Glu may be more strongly adsorbed to the material surface, thus prohibiting particle collapse; however, the Asp-based materials do not fully 
aggregate to bulk structures, indicating that the smaller acidic residue is important to prevent complete material aggregation. XRD analysis confirmed the CuS composition, which is important in understanding the reactivity of the materials, where the bandgap is well positioned for photocatalysis using visible light.

Once characterized, the two different CuS materials were subsequently probed for their photocatalytic reactivity in the degradation of three specific synthetic dyes: neutral red, methyl orange, and methylene blue. These specific dyes were selected because of their different charge states in solution: neutral (neutral red), anionic (methyl orange), or cationic (methylene blue). Dye degradation is a broadly used technique to quickly understand if a material can effectively harvest solar energy, as well as test the material's catalytic activity for potential environmental remediation applications. This is highly important as synthetic dyes are estimated to make up $20 \%$ of industrial water pollution. ${ }^{52}$ To drive photocatalytic degradation using a solar simulator, $\mathrm{H}_{2} \mathrm{O}_{2}$ was employed as a sacrificial oxidizing agent. For this reaction, the lyophilized $\mathrm{CuS}$ material was dispersed in an aqueous dye solution in a $1.0 \mathrm{~cm}$ quartz cuvette. This particle dye mixture was kept in the dark for $1.0 \mathrm{~h}$ under constant stirring to allow for dye surface adsorption to reach equilibrium. Upon reaching equilibrium, the $\mathrm{H}_{2} \mathrm{O}_{2}$ was added and the system was irradiated. The reaction was monitored for 90 min by UV-vis analysis of the decrease in dye absorbance due to degradation.

Fig. 8a presents a plot of the percent degradation of the dyes throughout the photocatalytic process employing the CuS materials prepared with Asp. In general, these materials degraded the neutral red dye the fastest, reaching completion in $\sim 40 \mathrm{~min}$. For anionic methyl orange and cationic methylene blue, $>97 \%$ of dye degradation was achieved within 90 min of irradiation. Similar degrees of reactivity were noted when the Glu-based CuS materials were employed as the catalysts, as shown in Fig. 8b. For this system, neutral red was again rapidly degraded within $\sim 40 \mathrm{~min}$; however, for the other dyes, slower reactivity was noted. To this end, $>97 \%$ of methyl orange was degraded within $90 \mathrm{~min}$, while methylene blue appeared to reach completion at $75 \mathrm{~min}$.

From the data of Fig. 8a and b, calculation of the pseudo first order rate constants $\left(k_{\text {obs }}\right)$ can be determined (Fig. $8 \mathrm{c}$ ). Note that the concentration of the $\mathrm{H}_{2} \mathrm{O}_{2}$ is in substantial excess to ensure that pseudo first order conditions are achieved. When considering the Asp-capped CuS materials, $k_{\text {obs }}$ values of $(7.4 \pm 1.2) \times$ $10^{-2},(3.8 \pm 1.2) \times 10^{-2}$, and $(2.1 \pm 0.8) \times 10^{-2} \mathrm{~min}^{-1}$ were observed for the degradation of neutral red, methyl orange, and methylene blue, respectively. For the Glu-based structures, similar $k_{\text {obs }}$ values were determined: $(8.2 \pm 0.8) \times 10^{-2} \mathrm{~min}^{-1}$ for neutral red, $(4.2 \pm 0.2) \times 10^{-2} \mathrm{~min}^{-1}$ for methyl orange, and $(3.0 \pm 0.7) \times 10^{-2} \mathrm{~min}^{-1}$ for methylene blue. As a control, the degradation of all dyes was processed under the same reaction conditions, but in the absence of CuS materials. In this situation, appreciable degradation only occurs with neutral red giving rise to a $k_{\text {obs }}$ of $(0.12 \pm 0.03) \times 10^{-2} \mathrm{~min}^{-1}$. Such a value is at least 61 times lower than the reaction driven by the CuScontaining reactions, confirming the reactivity of the inorganic materials.
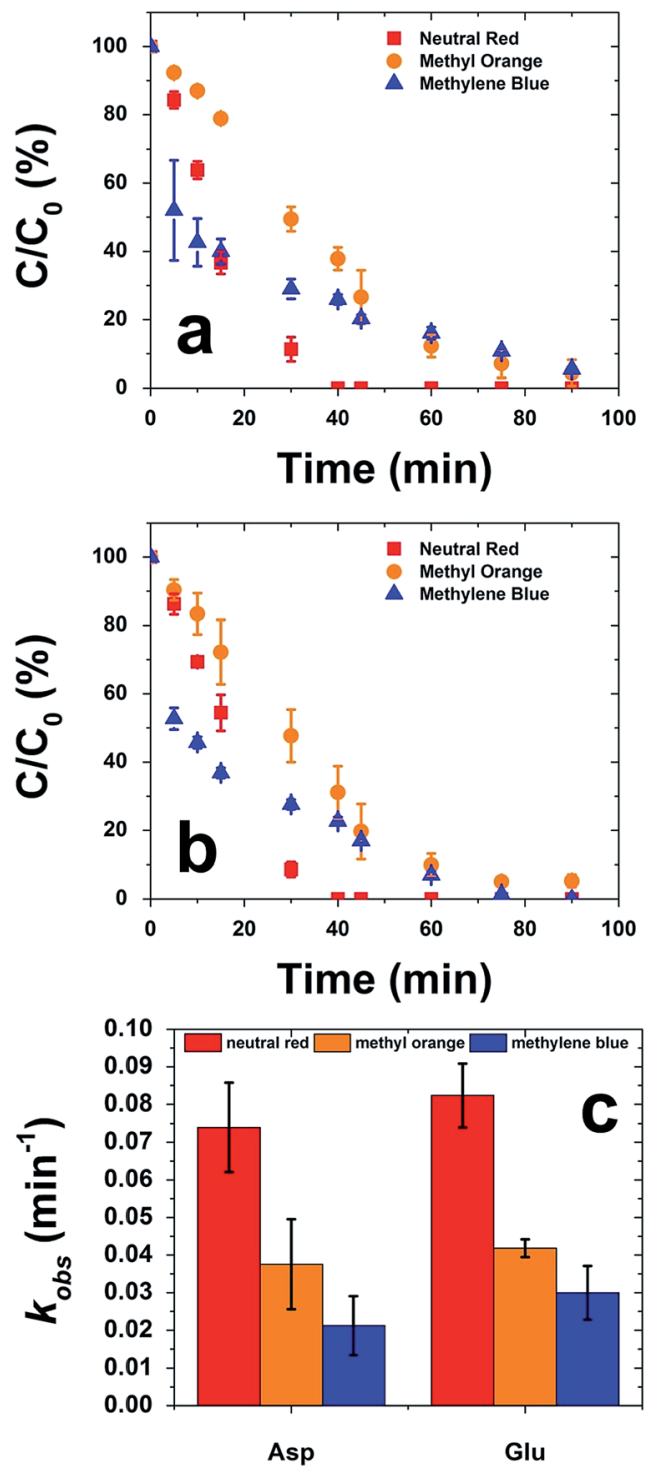

Fig. 8 Photocatalytic dye degradation analysis using the amino acidcapped CuS materials. Parts ( $a$ and $b$ ) present the degradation analysis for the (a) Asp- and (b) Glu-capped CuS materials, while part (c) display a comparison of the $k_{\text {obs }}$ values for each reaction.

For both material sets, the degradation of neutral red was substantially faster than the decomposition of the anionic methyl orange and cationic methylene blue dyes. Such effects suggest that charge on the dye molecule can substantially inhibit the reactivity due to electrostatic considerations at the particle surface. Thus, the neutral red molecule presents an advantage in photocatalysis. For both the methyl orange and methylene blue molecules, their individual charges are likely to alter the affinity of the dyes for the particle surface, which is negatively charged itself, potentially due to any bound acidic amino acids. This charge effect is anticipated to alter the interaction of the dyes for the CuS during the surface adsorption equilibration process in the dark, giving rise to the differences in observed photocatalytic reactivity. Such charge effects were also visually observed in the dispersion of the materials in 
the reaction medium; for the neutral red and methylene blue reactions, the CuS particles remained well dispersed, but in the degradation of methyl orange, formation of particle clumps at the top of the reaction system were evident. This suggests that electrostatics can substantially alter material dispersion and resultant reactivity, which is likely controlled by dye adsorption effects at the material surface.

Based upon the variety of reaction conditions and materials used in the literature, a direct comparison of the present materials to others is challenging. Differences in substrate identity and concentration relative to the concentrations of material and $\mathrm{H}_{2} \mathrm{O}_{2}$ used in the reaction, as well as adsorption times and light source all play significant roles in the final rates of degradation and total degradation time. For instance, $\mathrm{Xu}$ et al. employed a nanoporous CuS networked material as a catalyst for the degradation of Rhodamine B, methyl orange, and methylene blue. These materials were most reactive towards methylene blue with a maximum $k_{\text {obs }}$ value of $23.2 \times$ $10^{-2} \min ^{-1}$. While this system is comparable to the present catalytic system, Xu employs substantially higher concentrations of catalyst (4.9 times), peroxide (4.2 times), and dye substrate (34.8 times). ${ }^{23}$

Separate work by Tanveer et al. also exploited CuS prepared using solvothermal methods for the degradation of methylene blue using natural light instead of a solar simulator. In this work, two separate morphologies of CuS were examined: hollow microspheres ${ }^{53}$ and walled cuboctahedra. ${ }^{54}$ For the microsphere CuS materials, a maximal $k_{\text {obs }}$ of $2.4 \times 10^{-2} \mathrm{~min}^{-1}$ for methylene blue was observed; however, for the walled cuboctahedra, the supercomplex cuboctahedra of CuS demonstrated the greatest reactivity with a rate constant of $4.0 \times 10^{-2} \mathrm{~min}^{-1}$. Such values are similar in magnitude to the present materials for methylene blue degradation, but again differences in substrate ratios and the light source were present, making a direct comparison difficult. Additionally, it is important to note that for all the materials prepared by Tanveer et al., high reaction temperatures of $\geq 140{ }^{\circ} \mathrm{C}$ were employed, but for the amino acid directed synthesis, room temperatures are required to generate the catalytic materials.

Lastly, Yang et al. photodegraded methylene blue using the most similar amounts of $\mathrm{CuS}$, dye, and $\mathrm{H}_{2} \mathrm{O}_{2}$ as compared to the present system. For these studies, monodisperse CuS microflowers were made using a one-pot solvothermal synthesis with ethylenediamine and PVP at $160{ }^{\circ} \mathrm{C}$. With these materials, they are able to degrade $98 \%$ of the methylene blue in $25 \mathrm{~min}$, suggesting that the surface area of the materials is a key component in controlling the reaction; however, the synthetic conditions required high reaction temperatures over a long time period (12 h).

In general, the materials presented herein display substantial reactivity; however, it is important to understand their long term use for catalysis. Fig. 9 presents the recyclability analysis of the CuS materials over four photocatalytic cycles. Fig. 9a presents the analysis for neutral red, while Fig. 9b presents the results for the degradation of methylene blue. Note that due to lack of material dispersion in methyl orange, the recyclability analysis was not possible. For this study, the initial reaction was
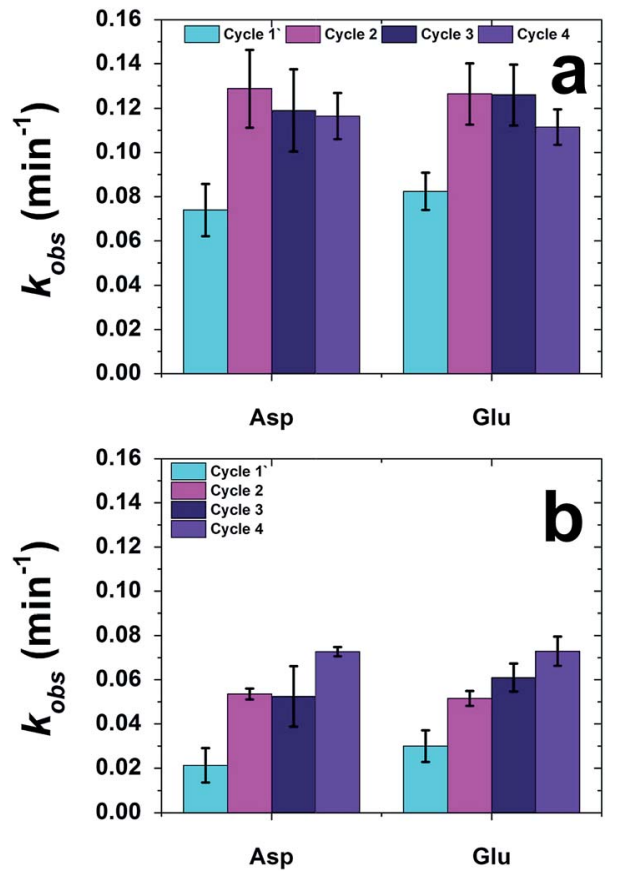

Fig. 9 Catalyst recyclability analysis for the photocatalytic degradation of (a) neutral red and (b) methylene blue.

processed as described above, and a subsequent irradiation (30 min) after the end of the degradation cycle was employed to eliminate extraneous material from the catalyst surface. Next, fresh dye and $\mathrm{H}_{2} \mathrm{O}_{2}$ was added to the reaction cuvette to reestablish the initial reaction conditions to maintain the first order kinetic analysis. Because there is likely residual $\mathrm{H}_{2} \mathrm{O}_{2}$ in the reaction from the previous cycle, the reaction is irradiated immediately upon the addition of the new reagents and the reaction is monitored.

For the recyclability analysis using neutral red, identical results were observed for both the Asp- and Glu-based materials (Fig. 9a). For the first cycle of the Asp-based CuS, a $k_{\text {obs }}$ value of $(7.4 \pm 1.2) \times 10^{-2} \mathrm{~min}^{-1}$ was noted that increased to $(12.9 \pm$ 1.8) $\times 10^{-2} \mathrm{~min}^{-1}$ for the second cycle. For all subsequent cycles, the $k$ values remain generally equivalent. Such effects were identical for the Glu-based materials using neutral red. This jump after the first reaction cycle may be the result of the changes in the equilibration of surface adsorption of the dye to the particle surface, which may change after the initial reaction cycle. Additionally, changes to the atomic structure of the CuS material at the particle surface may also be altered during the initial reaction cycle, as has been seen previously with different nanoparticle-driven catalytic reactions. ${ }^{55-57}$

When methylene blue was used for the recyclability analysis, increased reactivity as a function of reaction cycle was noted (Fig. 9b). For both the Asp- and Glu-based structures, the $k_{\text {obs }}$ values increased as a function of reaction cycle. Such changes in reactivity are likely due to changes in the surface equilibration and structure of the materials, as discussed for the neutral red recyclability analysis; however, the charged methylene blue may be more adversely altering these effects. Nevertheless, reactivity 
for both dyes over multiple catalytic cycles was observed, indicating that the materials are recyclable.

\section{Conclusions}

In conclusion, these results demonstrate that ambient, biomimetic synthetic pathways are available to produce CuS materials, which are growing in importance for a variety of applications. We found that acidic amino acids are able to passivate the growing metal sulfide materials in solution to generate nanodisk shaped structures. Interestingly, the dimensions of the structures changed as a function of the amino acid used in the synthesis, suggesting that the molecular structure is important in controlling the material structure. The as synthesized materials possessed a substantial plasmon band in the NIR; however, changes in the materials morphology were noted upon drying to the solid state. These structures were photocatalytically reactive using an $\mathrm{H}_{2} \mathrm{O}_{2}$-mediated Fenton-type reaction for the degradation of organic dyes. The process is highly recyclable and dependent upon the charge of the dye, suggesting that the surface morphology of the materials is critically important to the overall reactivity. The key result herein focuses on the fabrication of these unique materials under ambient, environmentally friendly, and sustainable conditions, which has proven to be challenging. The sustainable methods for CuS nanomaterial production demonstrated here may be important for a variety of applications from catalytic to biomedical.

\section{Conflicts of interest}

There are no conflicts to declare.

\section{Acknowledgements}

We greatly acknowledge the University of Miami for support. This material is based upon work supported by the Air Force Office of Scientific Research, Grant FA9550-18-1-0329. C. J. M. would also like to thank the University of Miami Dean's Dissertation Year Fellowship for support and the TEM Core at the University of Miami for microscopy analysis of our samples.

\section{Notes and references}

1 L.-J. Chen, S.-K. Sun, Y. Wang, C.-X. Yang, S.-Q. Wu and X.-P. Yan, ACS Appl. Mater. Interfaces, 2016, 8, 32667-32674. 2 N. Li, Q. Sun, Z. Yu, X. Gao, W. Pan, X. Wan and B. Tang, ACS Nano, 2018, 12, 5197-5206.

3 L. Wang, RSC Adv., 2016, 6, 82596-82615.

4 Y. Zhang, J. Tian, H. Li, L. Wang, X. Qin, A. M. Asiri, A. O. AlYoubi and X. Sun, Langmuir, 2012, 28, 12893-12900.

5 M. Dastkhoon, M. Ghaedi, A. Asfaram and H. Javadian, Appl. Organomet. Chem., 2018, 32, e4350.

6 A. Khan, S. J. Shah, K. Mehmood, Awais, N. Ali and H. Khan, J. Mater. Sci.: Mater. Electron., 2019, 30, 406.
7 H. Rao, W. Sun, S. Ye, W. Yan, Y. Li, H. Peng, Z. Liu, Z. Bian and C. Huang, ACS Appl. Mater. Interfaces, 2016, 8, 78007805 .

8 Y. Zhang, D. Wang, Q. Wang and W. Zheng, Mater. Des., 2018, 160, 870-875.

9 L. Tan, Z. Wu, X. Wang and J. Sun, RSC Adv., 2015, 5, 3531735324.

10 K. R. Nemade and S. A. Waghuley, Mater. Sci. Semicond. Process., 2015, 39, 781-785.

11 Y. Zhang, B. Deng, T. Zhang, D. Gao and A.-W. Xu, J. Phys. Chem. C, 2010, 114, 5073-5079.

12 D. Cornejo-Monroy, L. A. Martínez-Ortega, Z. Y. CastilloMorán, R. D. Molina-Arredondo, S. Noriega, V. TorresArgüelles and J. L. Enriquez-Carrejo, Appl. Phys. A: Mater. Sci. Process., 2017, 123, 547.

13 X. Liu, X. Wang, B. Zhou, W.-C. Law, A. N. Cartwright and M. T. Swihart, Adv. Funct. Mater., 2013, 23, 1256-1264.

14 Y. Liu, M. Liu and M. T. Swihart, J. Phys. Chem. C, 2017, 121, 13435-13447.

15 S. B. Aziz, M. A. Rasheed, S. R. Saeed and O. G. Abdullah, Int. J. Electrochem. Sci., 2017, 12, 3263-3274.

16 R. Kushwah, A. Singh, A. Anshul, D. Mishra and S. S. Amritphale, J. Mater. Sci.: Mater. Electron., 2017, 28, 5597-5602.

17 P. A. Ajibade and N. L. Botha, Nanomaterials, 2017, 7, 32.

18 M. Achimovicová, E. Dutková, E. Tóthová, Z. Bujnáková, J. Briančin and S. Kitazono, Front. Chem. Sci. Eng., 2019, 13, 164-170.

19 M. Pal, N. R. Mathews, E. Sanchez-Mora, U. Pal, F. ParaguayDelgado and X. Mathew, J. Nanopart. Res., 2015, 17, 301.

20 J.-H. Qin, Z.-Q. Liu, N. Li, Y.-B. Chen and D.-Y. Wang, J. Nanopart. Res., 2017, 19, 40.

21 S. Gorai, D. Ganguli and S. Chaudhuri, Cryst. Growth Des., 2005, 5, 875-877.

22 C. Wu, S.-H. Yu, S. Chen, G. Liu and B. Liu, J. Mater. Chem., 2006, 16, 3326-3331.

23 W. Xu, S. Zhu, Y. Liang, Z. Li, Z. Cui, X. Yang and A. Inoue, Sci. Rep., 2015, 5, 18125.

24 M. R. Knecht and T. R. Walsh, Bio-Inspired Nanotechnology, Springer-Verlag, New York, 2014.

25 T. R. Walsh and M. R. Knecht, Chem. Rev., 2017, 117, 1264112704.

26 N. A. Merrill, T. T. Nitka, E. M. McKee, K. C. Merino, L. F. Drummy, S. Lee, B. Reinhart, Y. Ren, C. J. Munro, S. Pylypenko, A. I. Frenkel, N. M. Bedford and M. R. Knecht, ACS Appl. Mater. Interfaces, 2017, 9, 8030-8040.

27 D. B. Pacardo, M. Sethi, S. E. Jones, R. R. Naik and M. R. Knecht, ACS Nano, 2009, 3, 1288-1296.

28 S. Chakraborty, V. R. Chelli, R. K. Das, A. S. Giri and A. K. Golder, Toxicol. Environ. Chem., 2017, 99, 434-447.

29 C. Song, M. G. Blaber, G. Zhao, P. Zhang, H. C. Fry, G. C. Schatz and N. L. Rosi, Nano Lett., 2013, 13, 3256-3261.

30 S. Mokashi-Punekar, A. D. Merg and N. L. Rosi, J. Am. Chem. Soc., 2017, 139, 15043-15048.

31 L. He, Y. Xiong, M. Zhao, X. Mao, Y. Liu, H. Zhao and Z. Tang, Chem.-Asian J., 2013, 8, 1765-1767. 
32 L. Shen, N. Bao, P. E. Prevelige and A. Gupta, J. Am. Chem. Soc., 2010, 132, 17354-17357.

33 E. D. Spoerke, B. A. Connor, D. V. Gough, B. B. McKenzie and G. D. Bachand, Part. Part. Syst. Charact., 2014, 31, 863-870.

34 J. P. da Costa, A. V. Girão, J. P. Lourenço, O. C. Monteiro, T. Trindade and M. C. Costa, J. Environ. Manage., 2013, 128, 226-232.

35 D. E. Ortiz-Ramos, A. I. Martínez-Enríquez and L. A. González, Mater. Sci. Semicond. Process., 2019, 89, 1825.

36 S. Banerjee, T. Maji, T. K. Paira and T. K. Mandal, Macromol. Rapid Commun., 2013, 34, 1480-1486.

37 R. A. McMillan, C. D. Paavola, J. Howard, S. L. Chan, N. J. Zaluzec and J. D. Trent, Nat. Mater., 2002, 1, 247.

38 H. Yan, J. Chen, Y. Li, Y. Bai, Y. Wu, Z. Sheng, L. Song, C. Liu and H. Zhang, Biomater. Sci., 2019, 7, 92-103.

39 R. Zhong, C. Peng, L. Chen, N. Yu, Z. Liu, M. Zhu, C. He and Z. Chen, RSC Adv., 2016, 6, 40480-40488.

40 O. Bretschger, A. Obraztsova, C. A. Sturm, I. S. Chang, Y. A. Gorby, S. B. Reed, D. E. Culley, C. L. Reardon, S. Barua, M. F. Romine, J. Zhou, A. S. Beliaev, R. Bouhenni, D. Saffarini, F. Mansfeld, B. H. Kim, J. K. Fredrickson and K. H. Nealson, Appl. Environ. Microbiol., 2007, 73, 7003-7012. 41 L. Han, Y. Zhang, X.-W. Chen, Y. Shu and J.-H. Wang, J. Mater. Chem. B, 2016, 4, 105-112.

42 Z. Wang, P. Huang, O. Jacobson, Z. Wang, Y. Liu, L. Lin, J. Lin, N. Lu, H. Zhang, R. Tian, G. Niu, G. Liu and X. Chen, ACS Nano, 2016, 10, 3453-3460.

43 Z. Mohammed Shahriar, M. Chung Hee, N. B. Krassimir and D. H. Elaine, Nanotechnology, 2013, 24, 325602.

44 V. Vinod Kumar, P. S. Hariharan, D. Eniyavan, N. Hari and S. P. Anthony, CrystEngComm, 2015, 17, 3452-3459.
45 S. B. Aziz, R. T. Abdulwahid, H. A. Rsaul and H. M. Ahmed, J. Mater. Sci.: Mater. Electron., 2016, 27, 4163-4171.

46 M. Liu, X. Xue, C. Ghosh, X. Liu, Y. Liu, E. P. Furlani, M. T. Swihart and P. N. Prasad, Chem. Mater., 2015, 27, 2584-2590.

47 X. Liu and M. T. Swihart, Chem. Soc. Rev., 2014, 43, 39083920.

48 W. Chen, Y. Xie, C. Hu, T. Zeng, H. Jiang, F. Qiao, J. Gu, X. Dong and X. Zhao, CrystEngComm, 2018, 20, 4283-4290.

49 Y. Xie, A. Riedinger, M. Prato, A. Casu, A. Genovese, P. Guardia, S. Sottini, C. Sangregorio, K. Miszta, S. Ghosh, T. Pellegrino and L. Manna, J. Am. Chem. Soc., 2013, 135, 17630-17637.

50 M. T. S. Nair, G. Laura and P. K. Nair, Semicond. Sci. Technol., 1998, 13, 1164.

51 S. M. M. Nelwamondo, M. J. Moloto, R. W. M. Krause and N. Moloto, Mater. Lett., 2012, 75, 161-164.

52 C. Coughlan, M. Ibáñez, O. Dobrozhan, A. Singh, A. Cabot and K. M. Ryan, Chem. Rev., 2017, 117, 5865-6109.

53 M. Tanveer, C. Cao, Z. Ali, I. Aslam, F. Idrees, W. S. Khan, F. K. But, M. Tahir and N. Mahmood, CrystEngComm, 2014, 16, 5290-5300.

54 M. Tanveer, C. Cao, I. Aslam, Z. Ali, F. Idrees, M. Tahir, W. S. Khan, F. K. Butt and A. Mahmood, RSC Adv., 2014, 4, 63447-63456.

55 Y. Zhang, Y. Xia, S. Yan, J. Han, Y. Chen, W. Zhai and Z. Gao, Dalton Trans., 2018, 47, 17461-17468.

56 A. Shivhare, S. J. Ambrose, H. Zhang, R. W. Purves and R. W. J. Scott, Chem. Commun., 2013, 49, 276-278.

57 S. Saha, A. Pal, S. Kundu, S. Basu and T. Pal, Langmuir, 2010, 26, 2885-2893. 